since they were all patients with a diagnosis of schizophrenia on a community mental health team's case-load.

The community mental health team concerned looked after an area of high socio-economic deprivation and the study included patients who had drifted down the social scale from more affluent rural areas where one would expect a lower prevalence of middle-ear disease. If there is any bias in this study it is likely to favour the null hypothesis rather than that suggested by Jainer \& Shivanandaswamy.

In addition, perhaps the most striking finding in this study was the excess of left-sided middle-ear disease. In this case, the odds ratio of 4.15 meets the recommendation of Sackett et al ${ }^{2}$ that an odds ratio of greater than 4 should be used to establish an association in case-control studies.

1 Mason P, Rimmer M, Richman A, Garg G, Johnson J, et al. Middle-ear disease and schizophrenia: case-control study. Br J Psychiatry 2008; 193: 192-6.

2 Sackett DL, Straus SE, Richardson WS, Rosenberg W, Haynes RB. EvidenceBased Medicine. How to Practice and Teach EBM. Churchill Livingstone, 2000.

Peter R. Mason, Cheshire and Wirral Partnership NHS Foundation Trust, The Stein Centre, St Catherine's Hospital, Derby Road, Birkenhead CH42 OLQ, UK. Email: peter.mason@cwp.nhs.uk

doi: 10.1192/bjp.194.1.89b

\section{DSM-V: should PTSD be in a class of its own?}

Principles of diagnostic taxonomy suggest that disorders of a specific class, or spectrum, should aggregate more with each other than with disorders from another class. Results of recent comorbidity studies raise questions about whether this is true for post-traumatic stress disorder (PTSD) - which has been classified as an anxiety disorder since DSM-III - and the implications for where the diagnosis should be located in DSM-V.

Several factor analyses of diagnostic data from epidemiological and clinical samples suggest that PTSD covaries more strongly with disorders defined by anhedonia, worry and rumination (i.e. the unipolar mood disorders and generalised anxiety disorder) than with ones characterised by pathological fear and avoidance (e.g. the phobias, panic/agoraphobia and obsessivecompulsive disorder). ${ }^{1}$ However, classifying PTSD among these 'anxious-misery' disorders provides a poor fit to the data because PTSD is conditional on trauma exposure and, in new-onset cases, typically develops before its comorbid conditions. For example, when PTSD and major depression co-occur following trauma exposure, PTSD usually precedes or develops concurrently with the depression. New-onset major depression that develops in the wake of trauma rarely precedes or develops in the absence of PTSD. ${ }^{2}$ This implies a causal influence of PTSD on comorbid psychopathology and suggests a distinct phenomenology which should be reflected in its diagnostic class membership within DSM.
Developmental studies have shown that adult psychopathology is often foreshadowed by childhood and/or adolescent problems in the same domain. Along these lines, many adults with anxiety disorders report histories of juvenile anxiety disorders but they do not typically report juvenile externalising disorders. The exception to this is found among samples of individuals with PTSD where adult patients frequently have histories of childhood externalising disorders. ${ }^{3}$ Twin studies align with this finding and have shown that PTSD shares genetic influences with both internalising- and externalising-spectrum diagnoses. ${ }^{4}$ Other work suggests that many adults with PTSD exhibit a predominantly externalising pattern of comorbidity characterised by problems in the domain of impulse-control, antisociality and substance misuse. ${ }^{5}$ These findings raise concern about conceptualising PTSD simply as the manifestation of a vulnerability to anxiety-related psychopathology.

Since its third edition, the DSM has taken a largely descriptive, as opposed to aetiological, approach to defining and classifying disorders. The most notable exception to this is the PTSD diagnosis, which specifies a causal relationship between trauma exposure and symptom development. We believe that the most appropriate location for PTSD in DSM-V would be among a class of disorders precipitated by serious adverse life events, i.e. a spectrum of traumatic stress disorders. Candidates for inclusion would include PTSD, acute stress disorder, adjustment disorder, a traumatic grief or bereavement-related diagnosis, and possibly complex PTSD. These disorders are the product of an environmental pathogen (i.e. a traumatic stressor) operating on individual diatheses that span the spectrum of human variation in vulnerability to psychopathology. This diathesis-stress interaction can result in extensive heterogeneity in the phenotypic expression of psychopathology - pathological anxiety being just one manifestation of the process.

1 Miller MW, Fogler JM, Wolf EJ, Kaloupek DG, Keane TM. The internalizing and externalizing structure of psychiatric comorbidity in combat veterans. J Trauma Stress 2008; 21: 58-65.

2 Breslau N, Davis GC, Peterson EL, Schultz LR. A second look at comorbidity in victims of trauma. The posttraumatic stress disorder-major depression connection. Biol Psychiatry 2000; 48: 902-9.

3 Gregory AM, Caspi A, Moffitt TE, Koenen K, Eley TC, Poulton R. Juvenile mental health histories of adults with anxiety disorders. Am J Psychiatry 2007; 164: 301-8

4 Koenen KC, Fu QJ, Lyons MJ, Toomey R, Goldberg J, Eisen SA, et al. Juvenile conduct disorder as a risk factor for trauma exposure and posttraumatic stress disorder. J Trauma Stress 2005; 18: 23-32.

5 Miller MW, Kaloupek DG, Dillon AL, Keane TM. Externalizing and internalizing subtypes of combat-related PTSD: a replication and extension using the PSY-5 scales. J Abnorm Psychol 2004; 113: 636-45.

Mark W. Miller, VA Boston Healthcare System, National Center for PTSD, USA. Email: mark.miller5@va.gov; Patricia A. Resick, Terence M. Keane, VA Boston Healthcare System, National Center for PTSD, USA

doi: 10.1192/bjp.194.1.90

\title{
Correction
}

Interventions for people bereaved through suicide: systematic review. BJP; 193: 438-43. Reference 5 should read: Hawton K, Simkin S. Helping people bereaved by suicide. Their needs require special attention. BMJ 2003; 327: 177-8. 